\title{
Spin and exchange coupling for Ti embedded in a surface dipolar network
}

\author{
Raghani Pushpa*1,2,4, Jesus Cruz ${ }^{3,2}$, and Barbara Jones ${ }^{2}$ \\ ${ }^{1}$ Center for Probing the Nanoscale, Stanford University, Stanford, California, USA \\ ${ }^{2}$ IBM, Almaden Research Center, San Jose, CA, USA \\ ${ }^{3}$ Georgetown University, Washington, D.C., USA and \\ ${ }^{4}$ Boise State University, Boise, Idaho, USA
}

(Dated: September 15, 2021)

\begin{abstract}
We have studied the spin and exchange coupling of $\mathrm{Ti}$ atoms on a $\mathrm{Cu}_{2} \mathrm{~N} / \mathrm{Cu}(100)$ surface using density functional theory. We find that individual Ti have a spin of 1.0 (i.e., 2 Bohr Magneton) on the $\mathrm{Cu}_{2} \mathrm{~N} / \mathrm{Cu}(100)$ surface instead of spin-1/2 as found by Scanning Tunneling Microscope. We suggest an explanation for this difference, a two-stage Kondo effect, which can be verified by experiments. By calculating the exchange coupling for Ti dimers on the $\mathrm{Cu}_{2} \mathrm{~N} / \mathrm{Cu}(100)$ surface, we find that the exchange coupling across a 'void' of $3.6 \AA$ is antiferromagnetic, whereas indirect (superexchange) coupling through a $\mathrm{N}$ atom is ferromagnetic. For a square lattice of $\mathrm{Ti}$ on $\mathrm{Cu}_{2} \mathrm{~N} / \mathrm{Cu}(100)$, we find a novel spin striped phase.
\end{abstract}

PACS numbers: 71.15.Mb, 71.70.Gm, 71.15.Nc, 68.55.-a,

\section{INTRODUCTION}

Atomic-scale magnetic structures on surfaces ${ }^{1}$ are of current interest for several reasons. Primarily, they display intriguing physical properties in their own right. Magnetic atoms on surfaces, simple or complex, can display large magnetocrystalline anisotropy which differs from the bulk. The spin can be large, or quenched by electronic effects such as the Kondo effect. And coupling between spins can be via direct overlap, RKKY or superexchange. Secondarily, these systems are compelling because of their parallels with other nano-scale systems - quantum dots, magnetic multilayers, magnetic impurities in thin films, to name just a few. Finally, there are the possible applications to nano-scale magnetic bits and future magnetic devices. A large net spin and magnetic anisotropy are required for atomic-scale magnetic structures to be used as practical nano-scale magnetic bits. A possible way to obtain a large magnetic moment is through a ferromagnetic coupling between transition metal atoms. However, ferromagnetic coupling is rare in transition metal complexes, ${ }^{2]}$ that is, when the transition metal atom is bonded to a nonmetallic atom. We describe below our studies of such a system.

In a Scanning Tunneling Microscope (STM) measurement ${ }^{3}$ of a $\mathrm{Ti}$ atom placed on a $\mathrm{Cu}_{2} \mathrm{~N} / \mathrm{Cu}(100)$ surface, it was found that the Ti exhibits very different magnetic properties than in gas phase. In the following work, we use density functional theory (DFT) to study the atomic spin of a single $\mathrm{Ti}$ atom, and exchange coupling of a dimer of $\mathrm{Ti}$ atoms, placed on a single layer of $\mathrm{Cu}_{2} \mathrm{~N}$ on a $\mathrm{Cu}(100)$ surface. The $\mathrm{Cu}_{2} \mathrm{~N}$ layer is used as an insulating layer to isolate the spin of a magnetic adatom from the metal electrons of the $\mathrm{Cu}(100)$ surface ${ }^{14}$. Hereafter, the $\mathrm{Cu}_{2} \mathrm{~N} / \mathrm{Cu}(100)$ surface will be referred to as the CuN surface. We study exchange coupling between $\mathrm{Ti}$ atoms in two different environments: (i) a square lattice of $\mathrm{Ti}$ on the $\mathrm{CuN}$ surface and (ii) a dimer of $\mathrm{Ti}$ atoms deposited on the
CuN surface.

\section{DFT CALCULATIONS}

We use spin-polarized DFT, as implemented in Quantum-ESPRESSO ${ }^{5}$, within a pseudopotential formalism using a plane wave basis with a cut-off of $30 \mathrm{Ry}$. A higher cut-off of $240 \mathrm{Ry}$ was used for the augmentation charges introduced by the ultrasoft pseudopotentia ${ }^{6}$. We use the generalized gradient approximation (GGA) for the exchange correlation interaction with the functional proposed by Perdew, Burke and Ernzerhof ${ }^{7}$ An on-site Coulomb interaction (U) for $\mathrm{Ti}$ was employed, with $U$ taken to be $4.7 \mathrm{eV}$ as calculated using a constraintGGA method 8 [9]. To improve the convergence, a gaussian smearing of width 0.01 Ry was adopted. Brillouin zone integrations for the $(1 \times 1)$ surface cell of $\mathrm{Cu}(100)$ were carried out using a $(16 \times 16 \times 1)$ mesh of $k$-points.

We obtained the bulk lattice parameter for $\mathrm{Cu}$ as 3.67 $\AA$, which compares well with the experimental value of $3.61 \AA^{10}$. To simulate the CuN surface, we use a symmetric slab of three to five atomic layers of $\mathrm{Cu}$, with a layer of $\mathrm{CuN}$ above and below. Periodic images of the slab were separated by a vacuum of $15 \AA$ along the $\mathrm{z}$ [100] direction.

\section{CUN SURFACE}

The top view of the CuN surface is shown in Fig. 17. The unit cell of the CuN surface (shown by the black square) consists of two $\mathrm{Cu}$ atoms and one $\mathrm{N}$ atom. We find that the $\mathrm{N}$ atoms are $0.18 \AA$ above the top $\mathrm{Cu}$ atoms in a fully relaxed structure. The distance between the first and second layers of $\mathrm{Cu}$ is $1.97 \AA$ which compares well with the all-electron result of $1.91 \AA 11$. We find that each $\mathrm{Cu}$ atom in the surface unit cell loses 0.7 electrons to the $\mathrm{N}$ (presumably due to the electronegative nature 


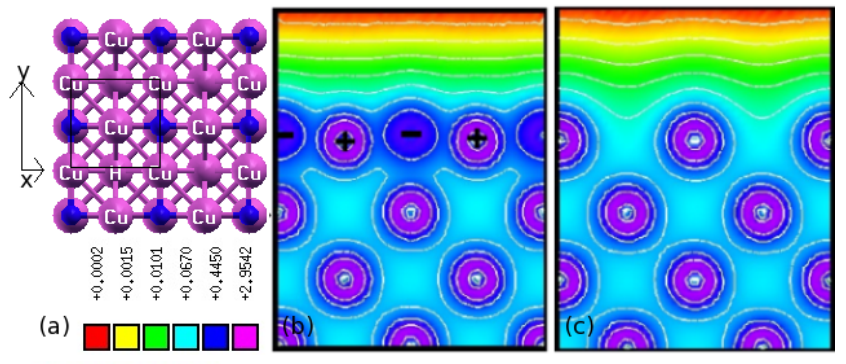

FIG. 1: (a) Top view of the $\mathrm{CuN}$ on $\mathrm{Cu}(100)$ surface; small and big spheres represent $\mathrm{N}$ and $\mathrm{Cu}$ atoms respectively. Big spheres with symbol "Cu" are the top layer $\mathrm{Cu}$ atoms and those without the symbol are the second layer. The black square indicates the $\mathrm{c}(1 \times 1)$ unit cell of the $\mathrm{CuN}$ surface. Along the $\mathrm{x}$-axis, surface $\mathrm{Cu}$ atoms are separated by a hollow site (it is marked by ' $\mathrm{H}$ ') and along the $\mathrm{y}$-axis, they are separated by a $\mathrm{N}$ atom. Side views of charge density produced by a cut along the (b) N-axis and (c) hollow-axis.

of $\mathrm{N}$ ). Thus, $\mathrm{Cu}$ and $\mathrm{N}$ atoms form a square network of dipoles on the surface, rendering a (nominally) insulating character to the surface. As shown in Fig:1a, along the $\mathrm{x}$-axis, two surface $\mathrm{Cu}$ atoms are separated by a hollow site and along the y-axis, they are separated by a $\mathrm{N}$ atom. These directions will be referred as hollow-axis and $\mathrm{N}$-axis, respectively. The charge density of the $\mathrm{CuN}$ surface along the N- and hollow-axis is shown in Fig, 1 (b) and (c) respectively. Notice that along the $\mathrm{N}$-axis, charge gets accumulated in the top layer.

\section{SPIN OF TI ON CUN SURFACE}

To calculate the spin of $\mathrm{Ti}$ on the $\mathrm{CuN}$ surface, a $\mathrm{Ti}$ atom is deposited on top of a $\mathrm{Cu}$ atom, following the STM experiments ${ }^{3}$. At one monolayer coverage of $\mathrm{Ti}$, the $(1 \times 1)$ surface unit cell consists of one $\mathrm{N}$ atom, one $\mathrm{Ti}$ atom and two $\mathrm{Cu}$ atoms as shown in Fig.1 1 a. A constrained-GGA ${ }^{[8]}$ calculation ${ }^{9}$ yields $U=4.7 \mathrm{eV}$ for $\mathrm{Ti}$ in this configuration. However, the value of $U$ for $\mathrm{Ti}$ would presumably be different at lower coverages of $\mathrm{Ti}$. In order to understand the effect of $\mathrm{U}$ on the spin of $\mathrm{Ti}$, we do calculations for a range of values of $U$ for the $(1 \times 1)$ unit cell. The results for distances between $\mathrm{Ti}$ and its nearest neighbor atoms in the surface, angle subtended by $\mathrm{Ti}-\mathrm{N}-\mathrm{Ti}$, and the spin of Ti are shown in Table [I. We find that Ti-N and Ti-Cu distances increase as $U$ increases, with the net effect of a rising $\mathrm{Ti}$ and decreasing (becoming sharper) Ti-N-Ti angle. Most importantly, as $\mathrm{U}$ increases the spin of $\mathrm{Ti}$ approaches that of the gas phase value of 1 .

As an added complexity, we find that the initial magnetization of Ti affects the final calculated ground state, indicating a complex energy minimization landscape. Hence we try several initial magnetizations and take the final state corresponding to the lowest energy. For
$\mathrm{U}=4.7$, we show the data corresponding to two such optimized structures (S-I and S-II) obtained by varying the initial magnetization (see Table I). The optimized structure corresponding to S-I is the lowest energy structure (S-I is lower in energy than S-II by $0.3 \mathrm{eV}$ ), showing the spin of Ti to be 0.75 , indicating possible mixed valent behavior for a monolayer of $\mathrm{Ti}$.

Next, we calculate the spin of Ti in $(2 \times 2),(2 \times 3)$, and $(3 \times 3)$ unit cells, i.e., at coverages of $1 / 4,1 / 6$, and $1 / 9 \mathrm{ML}$ respectively. This data is shown in the middle panel of Table I. Comparable to the $(1 \times 1)$ unit cell, the distance of $\mathrm{Ti}$ from the $\mathrm{Cu}$ below in $(2 \times 2)$ is $2.58 \AA$. There is also a similar trend of Ti rising higher above the surface than $\mathrm{N}$, by an amount increasing with increasing U. Most importantly, it was found that in all the three cases of larger unit cells, the spin on $\mathrm{Ti}$ is 1 . At this point, we note a discrepancy with $\mathrm{STM}^{3}$ experiments, which see a spin- $1 / 2$ Kondo effect. We postulate a resolution of the issue with a two-stage Kondo effect, in which the spin is first compensated from spin- 1 to spin- $1 / 2$ at a higher temperature, and then seen as a spin- $1 / 2$ Kondo effect at the experimentally observed temperature.

\section{EXCHANGE COUPLING}

To calculate the exchange coupling, we assume a Heisenberg interaction $\left(H=J \mathbf{S}_{\mathbf{1}} \cdot \mathbf{S}_{\mathbf{2}}\right)$, and can relate the value of $\mathrm{J}$ to the energy difference between ferromagnetic and (Ising) antiferromagnetic configurations:

$$
2 S^{2} J=E_{\uparrow \uparrow}-E_{\uparrow \downarrow} \equiv \Delta E
$$

Here, $S$ is the magnitude of spin, and $J$ is the exchange coupling. $E_{\uparrow \uparrow}$ and $E_{\uparrow \downarrow}$ are the total energies calculated from DFT when the spins on the magnetic atoms point along the same direction and in opposite directions respectively. Note that Eq. 1 holds for all values of quantum spin. The relationship with $\mathrm{J}$ is valid for each $S^{z}$ always at full maximal or minimal value (Ising antiferromagnet; colinear spins); for the energy difference with a full quantum antiferromagnetic state, the term $2 S^{2} J$ would become $(2 S+1) S J$. In this paper, we will mainly concentrate on the energy difference between ferromagnetic (aligned) and antiferromagnetic (antialigned) configurations, rather than on the value of J. We calculate the exchange coupling for $\mathrm{Ti}$ lattices (1ML coverage of $\mathrm{Ti}$ ) and for two $\mathrm{Ti}$ atoms placed on the $\mathrm{CuN}$ surface in a large unit cell.

\section{A. Lattice of Ti atoms on CuN surface}

At one monolayer coverage of $\mathrm{Ti}$ on $\mathrm{CuN}$ surface, $\mathrm{Ti}$ forms a square "lattice" on the surface. In this case, the energy of the ferromagnetic configuration $\left(E_{F M}\right)$ is the total energy of the $(1 \times 1)$ unit cell since it contains only 


\begin{tabular}{|c|c|c|c|c|c|c|}
\hline System & Cell & $U$ & $d_{T i-N}$ & $d_{T i-C u}$ & $A_{T i-N-T i}$ & $S$ \\
\hline \multirow{3}{*}{ Single } & & 0.0 & 1.91 & 2.49 & 148.6 & 0.0 \\
Ti & $1 \times 1$ & 3.0 & 1.95 & 2.54 & 141.6 & 0.6 \\
Atom & & $4.7(\mathbf{S}-\mathbf{I})$ & $\mathbf{1 . 9 9}$ & $\mathbf{2 . 5 6}$ & $\mathbf{1 3 5 . 6}$ & $\mathbf{0 . 7 5}$ \\
& & 6.0 & 2.33 & 2.72 & 104.2 & 1.0 \\
\cline { 2 - 7 } & $2 \times 2$ & 4.7 & 2.07 & 2.58 & - & 1.0 \\
& $2 \times 3$ & 3.0 & 1.98 & 2.52 & - & 1.0 \\
& $3 \times 3$ & 6.0 & 2.13 & 2.66 & - & 1.0 \\
\hline \multirow{2}{*}{ Ti Dimer } & N-axis & 4.7 & 2.04 & 2.65 & 142.9 & 1.0 \\
& H-axis & 4.7 & 2.09 & 2.56 & - & 1.0 \\
\hline
\end{tabular}

TABLE I: The Ti-N bond length $\left(d_{T i-N}\right)$, the Ti-Cu bond length $\left(d_{T i-C u}\right)$, the Ti-N-Ti angle $\left(A_{T i-N-T i}\right)$ and the spin $S$ of the $\mathrm{Ti}$ atom on the $\mathrm{CuN}$ surface. The top panel shows these results as a function of Hubbard $U$ (in $\mathrm{eV})$ on $\mathrm{Ti}$, for a $(1 \times 1)$ unit cell. The middle panel shows these results for a single Ti atom in $(2 \times 2),(2 \times 3)$, and $(3 \times 3)$ unit cells. The bottom panel shows these results for a dimer of Ti adsorbed along the N- and hollow-axis, respectively. All the bond lengths are given in Angstroms.

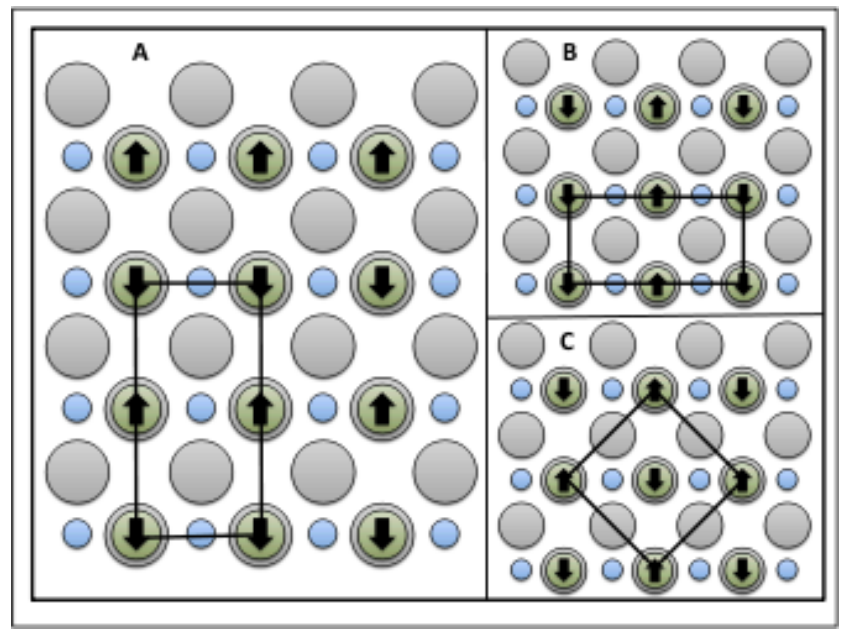

FIG. 2: Schematic diagrams showing spin configurations in Ti lattices. In configuration (a), spins are aligned along the $\mathrm{N}$-axis and antialigned along the hollow-axis; in (b) spins are antialigned along the $\mathrm{N}$-axis and aligned along the hollowaxis. Configuration (c) is a checkerboard configuration with spins antialigned along both the N- and hollow-axis.

one Ti atom. However, to obtain $E_{\uparrow \downarrow}$, we design three different configurations with $(1 \times 2),(2 \times 1)$, and $(\sqrt{2} \times \sqrt{2})$ unit cells as shown in Fig. 2(a), (b), and (c) respectively. Arrow signs in the figure indicate relative direction of spins on Ti atoms. Total energies of the three configurations will be referred to as $E_{H}, E_{N}$, and $E_{C}$ respectively. Notice that the unit cell size in all three configurations is twice that of the ferromagnetic configuration. Subtracting the total energies of configurations (a), (b), and (c) from two times the energy of the ferromagnetic configuration $\left(2 \times E_{F M}\right)$ will give the exchange coupling of $\mathrm{Ti}$ atoms along the hollow-axis, along the $\mathrm{N}$-axis, and in the checkerboard configuration, respectively; assuming that there are only nearest neighbor interactions.
Our results for exchange coupling are summarized in Table II For the lowest energy structure S-I, we find that the exchange coupling along the $\mathrm{N}$-axis is unexpectedly ferromagnetic, i.e., the total energy $E_{F M}$ is lower than $E_{N}$ by $16.1 \mathrm{meV}$. However, the exchange coupling across a hollow is antiferromagnetic, i.e., the total energy $E_{H}$ is lower than $E_{F M}$ by $106.8 \mathrm{meV}$. Thus, the antiferromagnetic coupling along the hollow-axis is much stronger than the ferromagnetic coupling along the $\mathrm{N}$-axis. The checkerboard pattern (Ising antiferromagnet) is more favored over a pure ferromagnetic state with $\Delta E$ being $77.8 \mathrm{eV}$; however, it is less favorable than the hollowaxis antiferromagnetism, presumably due to the energy disadvantage of antiferromagnetic coupling along the Naxis. The overall order, from lowest to highest energy, is $E_{H}<E_{C}<E_{F M}<E_{N}$. Configuration Fig. 2a is the ground state and we term it a spin striped state. These ferromagnetic stripes should be observable in large enough lattices.

In order to understand how structure plays a role in the exchange coupling, we also calculate spin exchange for the structure S-II (Table II). We notice that the exchange coupling for the structure S-II is much lower than that of S-I. This could possibly be due to lower interaction of $\mathrm{Ti}$ with the surface (See Table-I. Ti-Cu and Ti-N distances are longer in S-II than those in S-I). Spin density plots for the two structures in the ferromagnetic state are shown in Fig 3. Notice that the spin density gets stretched out along the hollow-axis for S-I. Also, the $\mathrm{N}$ atoms get spin polarized for S-I more than for S-II. This shows that in $\mathrm{S}-\mathrm{I}$, there are stronger interactions. The net result is that this structure has the lowest total energy.

\section{B. Dimer of Ti atoms on CuN surface}

We have drawn conclusions so far about Ti-Ti coupling based on the calculations in lattices, where the situation 


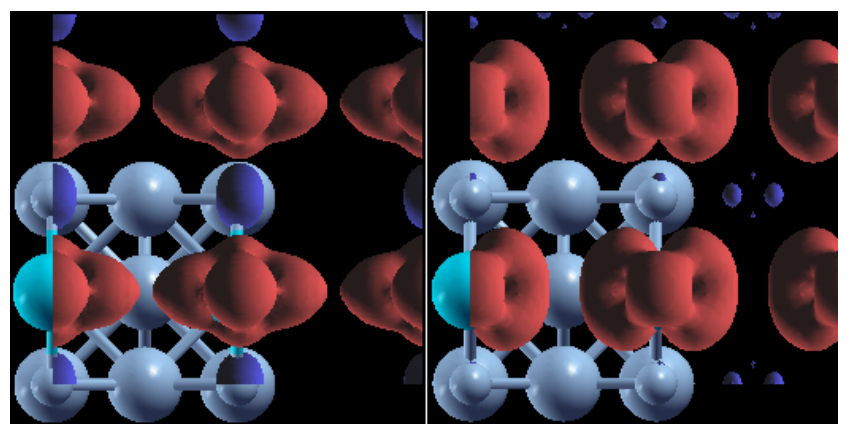

FIG. 3: Spin density plot for $(1 \times 1)$ unit cell for lower energy (a) and higher (b) configurations. Different colors (shadings in black and white version) correspond to opposite spins. The Nitrogen atoms appear as small spheres with opposite polarization between the $\mathrm{Ti}$.

\begin{tabular}{|c|c|c|c|}
\hline System & Structure & $E_{F M}-E_{N}$ & $E_{F M}-E_{H}$ \\
\hline \multirow{2}{*}{ Lattice $1 \times 1$} & S-I & -16.1 & 106.8 \\
& S-II & 6.2 & 13.9 \\
\hline Dimer $2 \times 3$ & - & -16.5 & 143.9 \\
\hline
\end{tabular}

TABLE II: The energy differences $\Delta E$ along the $\mathrm{N}$-axis $\left(E_{F M}-E_{N}\right)$ and the hollow-axis $\left(E_{F M}-E_{H}\right)$ for a lattice of $\mathrm{Ti}$ in a $(1 \times 1)$ unit cell, and a dimer of $\mathrm{Ti}$ in a $(2 \times 3)$ unit cell.

is more complicated because one not only has the nearest neighbor (NN) interactions but also has next NN (NNN) interactions and so on. To simulate a $\mathrm{Ti}-\mathrm{Ti}$ dimer on the surface we use a larger unit cell of $(2 \times 3)$ with two and three lattice units along the hollow- and N-axis respectively. Interestingly, we find ferromagnetic coupling along the $\mathrm{N}$-axis and antiferromagnetic along the hollowaxis, the same ground states as for the case of $(1 \times 1)$ lattices. Along the $\mathrm{N}$-axis, the energy difference $(\Delta E)$ is $-16.5 \mathrm{meV}$ compared to $-16.1 \mathrm{meV}$ for the $(1 \times 1)$ case. Along the hollow-axis the energy difference is $143.9 \mathrm{meV}$ compared to $106.8 \mathrm{meV}$ for the $(1 \times 1)$ case. Thus, a $\mathrm{Ti}$ lattice and a dimer show a similar trend and strength of coupling. It confirms our assumption of primarily nearest neighbor interactions in a Ti lattice on the $\mathrm{CuN}$ surface. Notice that the distance between $\mathrm{Ti}$ and the $\mathrm{Cu}$ atom below it is $2.56 \AA$ for both the $(1 \times 1)$ case, and the $(2 \times 3)$ case for coupling along the hollow-axis. However, when the dimer is placed along the $\mathrm{N}$-axis the distance between $\mathrm{Ti}$ and the $\mathrm{Cu}$ below it increases slightly to $2.65 \AA$. The Ti-N-Ti angle is 135.6 degrees for the $(1 \times 1)$ case which is close to 142.9 degrees for the $(2 \times 3)$ case.

In Fig. 4, we plot the spin density for the $\mathrm{Ti}$ dimer along the N-axis (Fig, $4 \mathrm{a}$ ) and the hollow-axis (Fig, $4 \mathrm{~b}$ ). A significant amount of induced spin-polarization around the $\mathrm{N}$ atom can be seen from the figure. Ferromagnetic coupling between $\mathrm{Ti}$ atoms along the $\mathrm{N}$-axis is established by having an opposite spin $\mathrm{N}$ atom both between the $\mathrm{Ti}$ atoms and at opposite ends. For anti-aligned spin configuration along the $\mathrm{N}$-axis, the $\mathrm{N}$ atom becomes a single- atom antiferromagnet with a net spin of zero. Along the hollow-axis, when spin on both the Ti atoms is aligned, a dramatic anisotropy in the spin polarization of the $\mathrm{Ti}$ develops, with a direct overlap established over the hollow site (Fig $4 \mathrm{~b}$ ). The stretching of the Ti bonds in this case case is striking, and suggestive that higher symmetry considerations may be coming into play. However, when spins are antialigned, no such elongation of spin polarization occurs. In both the cases, $\mathrm{N}$ atoms on the sides of the two $\mathrm{Ti}$ atoms develop a spin polarization opposite to that of the $\mathrm{Ti}$.

The primary sources of exchange coupling between the $\mathrm{Ti}$ atoms are superexchange ${ }^{12}$, RKKY ${ }^{13}$, and direct overlap/direct exchange $e^{10}$. The coupling between the adatoms can be direct, if the wave functions should overlap, or RKKY, if the influence of the $\mathrm{Cu}$ in the layers below is strong enough. Along the $\mathrm{N}$-axis, the center $\mathrm{N}$ atom becomes a natural source for a superexchange coupling between Ti atoms, ruling out RKKY which would need to take an indirect route under the $\mathrm{N}$ atom, a much longer route than directly across the $\mathrm{N}$ for superexchange. Along the hollow-axis, however, there is no convenient single atom to hop across for superexchange, rather the sea of conduction electrons from the underlying and intervening $\mathrm{Cu}$. (Unless one is to consider superexchange via the second-layer $\mathrm{Cu}$, an unlikely candidate.) In this case RKKY and direct overlap become more likely. Indeed for an aligned spin configuration, we observe a direct overlap forming, as discussed above. However, the lowest energy state for coupling along the hollow-axis is antiferromagnetic, and we conclude that in this case it is likely due to RKKY coupling. This could be tested experimentally by varying the $\mathrm{Ti}-\mathrm{Ti}$ distance and measuring the exchange coupling; however, only certain discrete lattice positions would be possible.

\section{CONCLUSIONS}

We find that a Ti atom has spin-1 on the $\mathrm{CuN}$ surface instead of spin- $1 / 2$ as found in the experiments 4 . As a possible explanation, we propose that there should be a two-stage Kondo effect for this system. At high temperatures, Ti starts with spin-1, which then undergoes a transition to spin-1/2 with a first-stage Kondo effect. At a lower temperature the second stage Kondo effect brings it down from spin- $1 / 2$ to a spin-zero, and it is this spin-1/2 Kondo effect that is seen in the low-temperature STM. This prediction can be tested by measuring the local density of states at higher temperatures than $0.5 \mathrm{~K}$.

We find a ferromagnetic coupling along the $\mathrm{N}$-axis and antiferromagnetic along the hollow-axis, for both the lattice and dimer of $\mathrm{Ti}$ on the $\mathrm{CuN}$ surface. Ti lattice and dimer have a similar trend as well as strength of coupling. This indicates that interactions between $\mathrm{Ti}$ atoms in the lattice configuration are local; and a marked spin striped phase is found as the ground state of the lattice. We find a ferromagnetic coupling along the $\mathrm{N}$-axis due to 


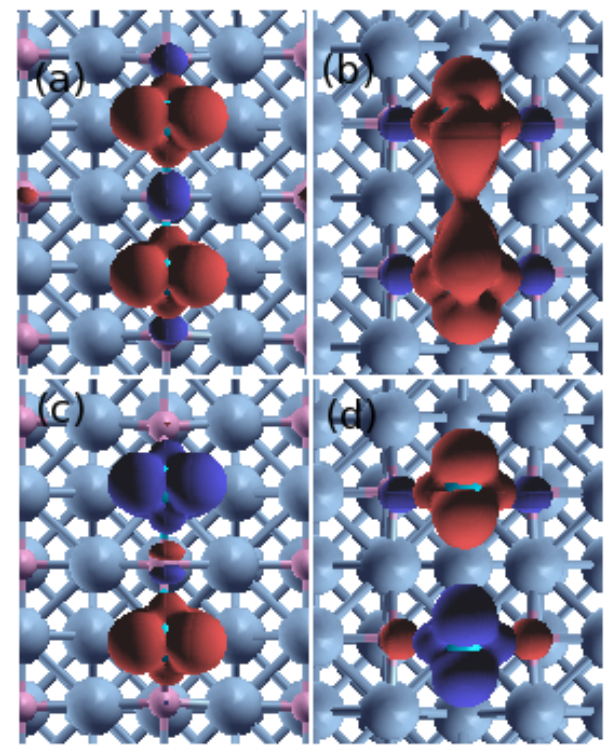

FIG. 4: Spin density $\left(\rho_{\uparrow}-\rho_{\downarrow}\right)$ plot for ferromagnetic (top pannel: (a) and (b)) and antiferromagnetic (bottom pannel: (c) and (d)) configuration of a Ti dimer along the (a) N-axis and (b) hollow-axis. The most energetically favorable configurations are ferromagnetic across a N (top left) and antiferromagnetic across a void (bottom right). superexchange, with secondary contributions from direct exchange. We also postulate that the antiferromagnetic coupling along the hollow-axis is primarily due to RKKY interactions, with a smaller direct exchange component.
1 C. F. Hirjibehedin, C. P. Lutz, and A. J. Heinrich, Science 312, 1021 (2006).

2 O. Kahn, Molecular Magnetism (VCH Publishers, 1993).

3 A. F. Otte, M. Ternes, K. von Bergmann, S. Loth, H. Brune, C. P. Lutz, C. F. Hirjibehedin, and A. J. Heinrich, Nature Physics 4, 847 (2008).

${ }^{4}$ C. F. Hirjibehedin, C.-Y. Lin, A. F. Otte, M. Ternes, C. P. Lutz, B. A. Jones, and A. J. Heinrich, Science 317, 1199 (2007).

5 S. Baroni, A. D. Corso, S. de Gironcoli, and P. Giannozzi (http://www.Quantum-Espresso.org, 2003).

6 D. Vanderbilt, Phys. Rev. B 41, 7892 (1990).

7 J. P. Perdew, K. Burke, and M. Ernzerhof, Phys. Rev. Lett. 77, 3865 (1996).
8 G.K.H.Madsen and P.Novák, Europhys. Lett. 69, 777 (2005).

9 t. b. p. C.-Y. Lin.

10 N. W. Ashcroft and N. D. Mermin, Solid State Physics (Saunders Company, 1976).

11 M. A. Barral, R. Weht, G. Lozano, and A. Llois, Physica B 398, 369 (2007).

12 A. Bencini and D. Gatteschi, EPR exchange coupled systems (Springer Verlag, 1990).

13 M. Ruderman and C. Kittel, Phys. Rev. 96, 99 (1954); T. Kasuya, Prog. Theor. Phys. 16, 45 (1956); K. Yosida, Phys. Rev. 106, 893 (1957). 